\title{
Hitting the complexity of the TIGIT-CD96-CD112R-CD226 axis for next-generation cancer immunotherapy
}

\author{
Hyung-seung Jin $^{1, *} \mathcal{E}$ Yoon Park ${ }^{2, *}$ \\ ${ }^{1}$ Department of Convergence Medicine, Asan Institute for Life Sciences, Asan Medical Center, University of Ulsan College of Medicine, \\ Seoul 05505, ${ }^{2}$ Theragnosis Research Center, Biomedical Research Division, Korea Institute of Science and Technology (KIST), Seoul \\ 02456, Korea
}

\begin{abstract}
Antibody-based therapeutics targeting the inhibitory receptors PD-1, PD-L1, or CTLA-4 have shown remarkable clinical progress on several cancers. However, most patients do not benefit from these therapies. Thus, many efforts are being made to identify new immune checkpoint receptor-ligand pathways that are alternative targets for cancer immunotherapies. Nectin and nectin-like molecules are widely expressed on several types of tumor cells and play regulatory roles in T- and NK-cell functions. TIGIT, CD226, CD96 and CD112R on lymphoid cells are a group of immunoglobulin superfamily receptors that interact with Nectin and nectin-like molecules with different affinities. These receptors transmit activating or inhibitory signals upon binding their cognate ligands to the immune cells. The integrated signals formed by their complex interactions contribute to regulating immune-cell functions. Several clinical trials are currently evaluating the efficacy of anti-TIGIT and anti-CD112R blockades for treating patients with solid tumors. However, many questions still need to be answered in order to fully understand the dynamics and functions of these receptor networks. This review addresses the rationale behind targeting TIGIT, CD226, CD96, and CD112R to regulate T- and NK-cell functions and discusses their potential application in cancer immunotherapy. [BMB Reports 2021; 54(1): 2-11]
\end{abstract}

\section{INTRODUCTION}

The immune system recognizes abnormal antigens on cancer cells and destroys them. The constant monitoring of emerging cancers by immune cells in the body is referred to as immune surveillance. The ability of tumors to escape surveillance is

*Corresponding authors. Hyung-seung Jin, Tel: +82-2-3010-0207; Fax: +82-2-3010-4103; E-mail: hsjin@amc.seoul.kr; Yoon Park, Tel: +822-958-5945; Fax: +82-2-958-5478; E-mail: ypark@kist.re.kr

https://doi.org/10.5483/BMBRep.2021.54.1.229

Received 6 October 2020, Revised 26 November 2020, Accepted 7 December 2020

Keywords: Cancer immunotherapy, CD112R, CD226, CD96, TIGIT important for their survival and propagation $(1,2)$. One mechanism by which tumor cells evade immune surveillance is activation of immune checkpoint inhibitory pathways. The immunecheckpoint receptors, such as cytotoxic T-lymphocyte-associated protein 4 (CTLA-4), and programmed cell-death protein 1 (PD-1), are expressed on $\mathrm{T}$ cells to limit their activation and effector functions. Antibody blockade targeting these receptors reinvigorates dysfunctional anti-tumor immunity and increases the objective response rate during treatment of some cancers. However, only a fraction of patients (10-30\%) benefits from immune checkpoint inhibitor (ICl) therapy (3-5). Therefore, it is pertinent to identify alternative approaches and targets that could lead to better response rates to immunotherapies. In addition, understanding the biology of novel immune checkpoint receptors and ligands would enable optimal clinical development of new drugs $(6,7)$.

TIGIT family receptors, a cluster of immunoglobulin superfamily receptors that interact with the Nectin and nectin-like molecules (Necls), has recently emerged as a potential cancer immunotherapy target $(8,9)$. These receptors include TIGIT (T-cell immunoglobulin and ITIM domain), CD226 (also known as DNAM-1, DNAX-associated molecule 1), CD96 (also known as TACTILE, T cell activation, increased late expression), and CD112R (also known as PVRIG, PVR-related Ig domain) (10). These receptors competitively or cooperatively interact with PVR (known as CD155 or Necl5), Nectin-1 (known as CD111 or PVRL1), Nectin-2 (known as CD1112 or PVRL2), Nectin-3 (known as CD113 or PVRL3), and/or Nectin-4 (known as PVRL4) (Table 1 and Fig. 1).

The complex interactions between these receptors and ligands determine the profile of immune cell activation $(11,12)$. The availability of ligands and the kinetics of cognate receptor expression could determine the activity of tumor infiltrating lymphocytes (TILs) depending on the context. Here, we review recent efforts to understand the function of the TIGIT family of receptors (TIGIT, CD226, CD96 and CD112R) in cancer immunology, on which future studies can be designed to address the potency of targeting these receptors in cancer immunotherapies. 
Table 1. Nectin and nectin-like molecules that interact with TIGIT family receptors

\begin{tabular}{lll}
\hline Member & \multicolumn{1}{c}{ Alias } & \multicolumn{1}{c}{ Interacting partners } \\
\hline PVR & CD155, Necl-5 & TIGIT, CD226, CD96 \\
Nectin-1 & CD111, PVRL & CD96 \\
Nectin-2 & CD112, PVRL2 & TIGIT, CD226, CD112R \\
Nectin-3 & CD113, PVRL3 & TIGIT \\
Nectin-4 & PVRL4 & TIGIT \\
\hline
\end{tabular}

\section{THE LIGANDS FOR THE TIGIT FAMILY OF RECEPTORS: NECTIN AND NECTIN-LIKE MOLECULES}

Nectin and Necl proteins are cell-adhesion molecules that belong to the immunoglobulin (Ig) superfamily. Nectins are a family consisting of four members (Nectin-1-4), and the Necl family consists of five members (Necl-1-5). They are type-I glycoproteins containing an extracellular region with multiple immunoglobulin domains that mediate various trans and cis interactions between Nectins/Necls and their family members and with other surface proteins. Some Nectins or Necls are highly upregulated in several human malignancies and have gained much attention as immune regulators in cancer immunology (13-15).

PVR is upregulated in various types of tumors and is involved in mediating tumor cell invasion and migration (16). PVR expression is associated with an unfavorable prognosis across various solid cancer types $(17,18)$. PVR also plays immunoregulatory roles by interacting with three different receptors, which are CD226, TIGIT, and CD96, expressed on T and NK cells (19-21). TIGIT binds to PVR with the highest affinity, whereas CD96 and CD226 has intermediate and the lowest affinity for PVR binding, respectively. Inhibitory signaling to immune cells might be mediated through competition between TIGIT and other receptors, such as CD226 and CD96, for PVR (22-24), which is expressed in a soluble form by alternative splicing in humans. High levels of soluble PVR (sPVR) have been detected in several human cancers. A recent report by Okumura $G$ et al. has demonstrated that SPVR inhibits CD226-mediated cytotoxic activity of NK cells in a mouse melanoma model (25).

Nectin-2 is expressed on tumor and tumor-infiltrating myeloid cells. Like PVR, Nectin-2 binds to CD226 or TIGIT expressed on T and NK cells, leading to anti-tumoral or pro-tumoral immune responses, respectively. However, both CD226 and TIGIT have much weaker affinity to Nectin-2 than to PVR. A structural and biophysical analysis showed that TIGIT binding disrupts pre-assembled Nectin-2 oligomers (26). CD96 does not interact with Nectin-2. CD112R binds only to Nectin-2 with high affinity and suppresses T-cell function $(22,27,28)$. Whelan et al. have demonstrated that the inhibitory effect of Nectin-2 is mediated by CD112R but not by TIGIT. They showed that

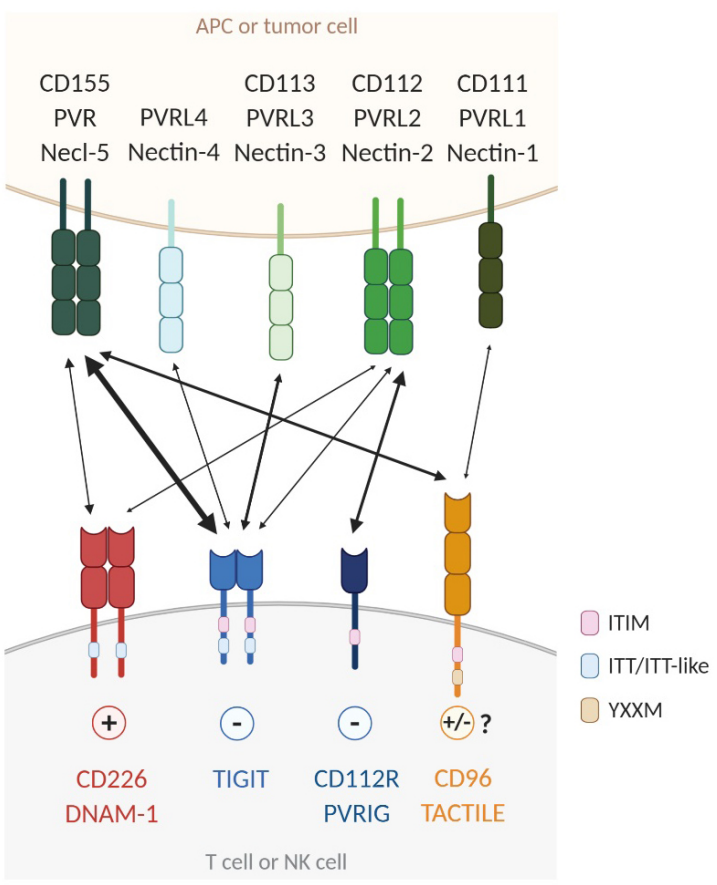

Fig. 1. Complex interactions of TIGIT family receptors with Nectin and nectin-like molecules. TIGIT, CD226, CD96, and CD112R are mainly expressed on activated T cells and NK cells. Their ligands, PVR, Nectin-1, Nectin-2, Nectin-3 and Nectin-4, are expressed on tumor cells and antigen-presenting cells (APCs). TIGIT and CD112R deliver inhibitory signals through ITIM motifs in their cytoplasmic domain. The ITT-like motif of TIGIT also plays a role in its negative signaling. CD226 delivers activating signals through an ITT/ITTlike motif. Both human and mouse CD96 sequences contain an ITIM motif, but human CD96 also contains an YXXM motif. CD96 signaling induces immunosuppression in mouse $\mathrm{T}$ and NK cells, but whether this receptor in human triggers inhibitory or activating signaling needs to be clarified. CD112R delivers an inhibitory signal through its ITIM motif. TIGIT binds to PVR, Nectin-2, Nectin-3 and Nectin-4. CD226 interacts with PVR and Nectin-2. CD96 binds PVR and Nectin-1. CD226 competes with both TIGIT and CD96 for PVR engagement and with CD112R for Nectin-2 binding. TIGIT has a greater affinity for PVR than CD226 and CD96. The interaction of Nectin-2 with CD112R is of higher affinity than with CD226. Interactions between receptors and ligands are depicted by two-sided arrows. The arrows are proportional to the reported affinities of the interactions.

blocking the Nectin-2-CD112R axis increases effector T-cell function in cancer, suggesting that the Nectin-2-CD112R and PVR-TIGIT pathways could be nonredundant inhibitory signaling pathways (10).

Nectin-1 has been identified as a CD96 ligand. The affinity of human CD96 for Nectin-1 is lower than that for PVR (29). Nectin-3 interacts with TIGIT. Nectin-3 expression is restricted to non-hematopoietic tissues (30). Nectin-4 has recently been reported as a ligand that interacts with TIGIT alone. Nectin- 4 expression is restricted to cancer cells, and Nectin- 4 blockade 
improves the NK cell-mediated antitumor response in mice (31).

\section{TIGIT FAMILY OF RECEPTORS}

\section{TIGIT}

TIGIT is an inhibitory Ig receptor expressed by effector and memory $\mathrm{CD}^{+} \mathrm{T}$ and $\mathrm{CD} 8^{+} \mathrm{T}$ cells, regulatory $\mathrm{T}$ cells $\left(\mathrm{T}_{\text {regs }}\right.$ ), follicular T helper cells, and NK cells. The cytoplasmic tail of TIGIT contains an immunoreceptor tyrosine-based inhibitory motif (ITIM) and an immunoglobulin tail tyrosine (ITT)-like motif, which initiate an inhibitory signaling cascade. TIGIT has multiple binding partners, including PVR, Nectin-2, Nectin-3, and Nectin-4 (32). Knockdown of TIGIT expression in human $\mathrm{CD}^{+} \mathrm{T}$ cells increases the expression of T-bet and interferon (IFN)- $\gamma$, which is overcome by blocking CD226 signaling, suggesting that TIGIT inhibits T cells by competing with CD226 for binding to the same PVR ligand (33). In another mechanism suggesting TIGIT interference with CD226-mediated costimulation, TIGIT binds to CD226 in the cis position and prevents its homodimerization in T cells (34). TIGIT knockout mice do not develop spontaneous signs of autoimmunity but develop more severe experimental autoimmune encephalitis than do wild-type mice when immunized with the myelin oligodendrocyte glycoprotein, indicating a suppressive role for TIGIT in T cells (32). TIGIT is highly expressed by a subset of $\mathrm{T}_{\text {reg }}$ cells and is associated with a more suppressive phenotype. TIGIT-expressing $T_{\text {reg }}$ subsets specifically suppress proinflammatory $\mathrm{T}$ helper 1 (Th1) and Th17 cells, but not Th2-type T-cell responses. TIGIT activation in $\mathrm{T}_{\text {reg }}$ cells leads to T-cell suppression by producing interleukin (IL)-10 and fibrinogen-like protein $2(35,36)$.

TIGIT expression and clinical outcomes in cancer: Increased TIGIT expression on TILs has been observed in various human cancers, including non-small-cell lung carcinoma (NSCLC), melanoma, head and neck squamous cell carcinoma (HNSCC), colorectal cancer (CRC), glioblastoma (GBM), gastric cancer, liver cancer, multiple myeloma (MM), acute myeloid leukemia (AML), and follicular lymphoma (FL) (15, 37-47). TIGIT-expressing CD8 ${ }^{+}$TILs are most likely in an exhausted state characterized by high co-expression of inhibitory immune checkpoint receptors, such as PD-1, lymphocyte-activation gene 3 (LAG-3), T-cell immunoglobulin, and mucin-domain containing-3 (TIM-3), and have an impaired capacity to proliferate and produce cytokines $(34,48-50)$. Several immune monitoring studies in cancer patients have reported functional defects of $\mathrm{TIGIT}^{+} \mathrm{CD}^{+}$ $\mathrm{T}$ cells and associated clinical outcomes. Bone marrow $\mathrm{CD} 8^{+} \mathrm{T}$ cells with a high frequency of TIGIT expression are less responsive to TCR/CD28 or NY-ESO-1 cancer testis antigen stimulation than are $\mathrm{TIGIT}^{-} \mathrm{CD}^{+} \mathrm{T}$ cells in MM patients (40). $\mathrm{CD}^{+} \mathrm{T}$ cells from the peripheral blood of AML patients express a high level of TIGIT, and $\mathrm{TIGIT}^{+} \mathrm{CD}^{+} \mathrm{T}$ cells show functional defects in cytokine production and survival, which are restored by TIGIT knockdown. Increased frequency of $\mathrm{TIGIT}^{+} \mathrm{CD}^{+} \mathrm{T}$ cells is correlated with $\mathrm{AML}$ relapse and post-allogeneic stem-cell transplantation progression (38). A similar observation was reported in a study of gastric cancer patients. $\mathrm{TIGIT}^{+} \mathrm{CD}{ }^{+} \mathrm{T}$ cells from peripheral blood exhibited functional exhaustion, with reduced proliferation, cytokine production, and glucose uptake upon co-culture with PVR-expressing gastric cancer cells, which was restored by adding exogenous glucose or inhibiting PVRTIGIT signaling (41). TIGIT-mediated immune dysfunction of $\mathrm{CD}^{+} \mathrm{T}$ cells is associated with recurrence in patients with gastric cancer (51). A recent study reported that the abundance of intratumoral $\mathrm{TIGIT}^{+} \mathrm{T}$ cells in $\mathrm{FL}$ is correlated with unfavorable patient outcomes and poor survival (37). TIGIT is also highly expressed on tumor-infiltrating $\mathrm{T}_{\text {reg }}$ cells. TIGIT expression confers stability to the lineage and increases suppressive capacity in both mouse and human $\mathrm{T}_{\text {reg }}$ cells $(35,36,52)$. Increased TIGIT expression on tumor-infiltrating $\mathrm{T}_{\text {reg }}$ cells with an activated phenotype and highly suppressive activity is correlated with poor clinical outcomes in patients with $\mathrm{FL}$, hepatocellular carcinoma (HCC) and metastatic melanoma (37, 53, 54).

Anti-tumor efficacy of a TIGIT blockade: Emerging evidence has provided opportunities for therapeutic interventions targeting TIGIT with antagonistic monoclonal antibodies (mAbs), which block ligand binding, including PVR and Nectin-2. Treatment with anti-TIGIT antagonist mAbs as a single agent does not induce sufficient tumor regression in MC38 colon carcinoma, $\mathrm{CT} 26$ colon carcinoma, or $\operatorname{Trp} 53^{\mathrm{KO}} / \mathrm{C}-\mathrm{Myc} \mathrm{CE}^{\mathrm{OE}} \mathrm{HCC}$ mouse tumor models $(34,55,56)$, whereas reduced tumor burden and increased survival are observed in mouse myeloma (Vk12653 and Vk12598) or the Tgfbr1/Pten2 CKO HNSCC mouse model after TIGIT blockade $(40,46)$. These discrepancies may be attributed to the different characteristics of each tumor type, such as 1) ligand expression levels, with different sensitivities to TIGIT blockade controlled by PVR and PD-L1 expression (57); or 2) the tumor microenvironment (TME), which has elevated TIGIT engagement to PVR in an acidic $\mathrm{pH}$ environment (58). A TIGIT blockade elicits an antitumor effect mainly by promoting $\mathrm{CD}^{+} \mathrm{T}$ cell or inhibiting $\mathrm{T}_{\text {reg }}$ cell responses (34, $40,46)$. However, a recent study by Zhang et al. proposed the NK-cell-dependent therapeutic efficacy of TIGIT blockade. Anti-TIGIT mAb treatment at an early time point (3 days after subcutaneous implantation of tumor cells) inhibited tumor growth in CT26 or methylcholanthrene (MCA)-induced fibrocarcinomabearing mice by preventing exhaustion of tumor-infiltrating NK cells, which resulted in an increased antitumor response of $\mathrm{CD}^{+} \mathrm{T}$ cells (15). Yet the mechanisms used by NK cells to boost $\mathrm{CD}^{+} \mathrm{T}$ cell function after TIGIT blockade are unclear and need to be further elucidated. Combined blockade of TIGIT and PD-1/PD-L1 had potent antitumor efficacy in several subcutaneous tumor models, including MC38, CT26, and EMT6, where TIGIT or PD-1/PD-L1 blockade alone had limited efficacy $(34,55)$. Recent studies using orthotopic mouse models of HCC or glioblastoma reported that dual blockade of TIGIT and PD-1 substantially improves tumor regression and long- 
term survival of tumor-bearing mice by promoting effector functions of $\mathrm{CD}^{+}{ }^{+} \mathrm{T}$ cells and anti-tumor immunologic memory responses $(56,59)$. Another approach to combining TIGIT blockade with other therapies showed that a triple combination treatment of anti-TIGIT mAb, anti-PD-L1 mAb, and radiotherapy resulted in almost complete tumor regression in CT26-bearing mice (60). Accumulating data indicate that TIGIT blockade reinvigorates the T-cell response in cancer patients. Human anti-TIGIT $\mathrm{mAb}$ and/or anti-PD-1 mAb treatment increases proliferation and cytokine production of NY-ESO-1 specific $\mathrm{CD}^{+} \mathrm{T}$ cells upon stimulation by the NY-ESO- $1^{157-165}$ peptide in peripheral blood of melanoma patients; this effect was further confirmed in CD8 ${ }^{+}$TILs from metastatic melanoma patients that exhibited an increased capacity for proliferation and degranulation in response to TIGIT and/or PD-1 blockade (39). Inhibiting TIGIT by blocking the mAb improves cytokine production by bone-marrow $\mathrm{CD}^{+} \mathrm{T}$ cells in $\mathrm{MM}$ patients upon stimulation with anti-CD2/anti-CD3/anti-CD28 microbeads (40). More recently, Jin HS et al. showed that TIGIT blockade promotes CEF (CMV, EBV, flu) peptide antigen-specific proliferation and IFN- $\gamma$ secretion of peripheral-blood memory $\mathrm{CD}^{+} \mathrm{T}$ cells obtained from pancreatic ductal adenocarcinoma (PDAC) patients after mFOLFIRINOX therapy (48).

Mode of action of anti-TIGIT therapy: It has been proposed that TIGIT exerts its immunosuppressive effects by outcompeting CD226 for PVR binding $(20,34)$. However, the molecular interplay between TIGIT, CD226, and PVR remains unclear, but is particularly important for understanding the mode of action of anti-TIGIT therapy that contributes to its clinical success. One study suggested that TIGIT acts as a decoy receptor to indirectly affect CD226 activation by binding to PVR, and SHP2 recruited by PD-1 dephosphorylates CD226 (61). Moreover, GITR agonism in response to anti-GITR mAb treatment induces downregulation of TIGIT on CD8 ${ }^{+}$TILs, although the molecular network between TIGIT and GITR was not investigated. The functional association between CD226 signaling and the PD1-SHP2 pathway has been shown in MC38 or RENCA (kidney carcinoma) tumor models where CD226 blockade reversed the antitumor response induced by a combined anti-PD-1 and anti-GITR mAb treatment. This study proposed a novel molecular mechanism for TIGIT-CD226 axis regulation, but it is still unclear how the PD-1-SHP2 axis integrates into the PVR-CD226 signaling pathway. In addition, the role of TIGIT ITT-like and ITIM motifs, which recruit SHP1 upon ligation of PVR to inhibit PI3K and MAPK signaling in NK cells (62) in T-cell regulation, needs to be further elucidated. Jin HS et al. demonstrated the direct effect of TIGIT on intracellular regulation of CD226 activation in response to PVR binding (48). Jin $\mathrm{HS}$ et al. generated a specific antibody against the CD226 tyrosine 322 and detected reduced tyrosine phosphorylation of CD226 in Jurkat cells expressing the TIGIT wild type, but not tyrosine mutations at the ITT-like and ITIM motifs of TIGIT (TIGIT ${ }^{\text {Y225AY231A }}$ ). This impaired CD226 phosphorylation/activation was restored by anti-TIGIT blocking mAb treatment, suggesting that TIGIT blockade depends on CD226 tyrosine phosphorylation. The effect of TIGIT blockade was observed only in human $\mathrm{CD} 8{ }^{+} \mathrm{T}$ cells expressing the CD226 wild type or the CD226 mutation at serine 329 (S329A) but not the tyrosine mutation at 322 (Y322A) in the presence of PVR. This was the first study to find out the mode of action of anti-TIGIT blocking mAbs that could provide a mechanism-based rationale for designing an optimal clinical strategy for anti-TIGIT therapy as well as in combination with other cancer therapies.

\section{CD226}

CD226 is a co-stimulatory receptor that is expressed by CD4 ${ }^{+}$ and $\mathrm{CD}^{+}{ }^{\top} \mathrm{T}$ cells, $\gamma \delta \mathrm{T}$ cells, NK cells, monocytes, and a small population of B cells. CD226 interacts in the cis position with lymphocyte function-associated antigen-1 (LFA-1) to promote cell adhesion and transduce T-cell activation signaling (63, 64). CD226 also binds to PVR and Nectin-2, which leads to a cytotoxic immune response against a range of tumor cells (65). CD226 plays a crucial role in the formation of the immunological synapse (IS), and CD226 deficiency in mouse $C D 8^{+} T$ cells and NK cells have IS defects, leading to impaired antitumor immunity. CD226 deficient mice display a greater tumor burden to a variety of tumors than do wild-type mice (66).

CD226 expression and clinical cancer outcomes: Studies in mice and humans have reported decreased CD226 expression in TILs $(19,39,48,67,68)$. In 4T1 mammary carcinoma, B16F10 melanoma, CT26 or MC38-bearing mice, CD44 ${ }^{+} \mathrm{CD} 8^{+}$ T cells expressing a low level of CD226 accumulate at the tumor site, while splenic $\mathrm{CD} 44^{+} \mathrm{CD} 8{ }^{+} \mathrm{T}$ cells display high CD226 expression (48). CD226 ${ }^{\mathrm{lo}} \mathrm{CD}^{+}$TILs exhibit an exhausted phenotype with upregulation of TIGIT, PD-1, Tim-3, Lag-3, CD101, CD38, and Eomes, and reduced expression of CD127, Slamf6, and T-bet. Consistent with the exhausted phenotype, a functional impairment was found in $\mathrm{CD} 226^{\mathrm{lo}} \mathrm{CD} 8^{+}$ TILs with attenuated polyfunctionality and proliferative capacity compared to CD226 $6^{\text {hi }} \mathrm{CD}^{+}$TILs isolated from $4 \mathrm{~T} 1$ or MC38 tumor-bearing mice (48). Downregulation of CD226 and the associated exhaustion phenotype were also observed in bone-marrow (BM) CD8 ${ }^{+} \mathrm{T}$ cells from Vk12653 MM-bearing mice that had relapsed after autologous stem-cell transplantation, but MM-controlled mice retained a high level of CD226 expression in the BM (68). Moreover, the number of BM $\mathrm{CD}^{+} \mathrm{T}$ cells expressing CD107a and IFN- $\gamma$ was inversely correlated with myeloma burden in MM-relapsed mice. The imbalance between CD226 and TIGIT expression on CD8 ${ }^{+}$TILs has also been observed in patients with melanoma, RCC, CRC, and NSCLC. NY-ESO-1-specific CD8 ${ }^{+}$TILs, but not circulating $\mathrm{CD}^{+} \mathrm{T}$ cells, display less CD226 expression with high TIGIT and PD-1 expression in metastatic melanoma patients (39). A recent study further delineated the characteristics of $\mathrm{CD} 226^{\mathrm{lo}} \mathrm{CD}^{+}$ T cells in patients with RCC, CRC, or NSCLC, as well as in healthy donors (48).

1) $\mathrm{CD} 226^{\mathrm{l}} \mathrm{CD}^{+}{ }^{+}$TILs express high levels of TIGIT, PD-1, Tim-3, and Lag-3; 
2) Downregulation of CD226 is associated with progressive differentiation of $\mathrm{CD}^{+} \mathrm{T}$ cells;

3) $\mathrm{CD} 226^{\mathrm{lo}} \mathrm{CD} 8{ }^{+} \mathrm{T}$ cells exhibit poor responsiveness to antigen-specific stimulation.

In line with the reduced CD226 expression in exhausted/ dysfunctional $\mathrm{CD}^{+} \mathrm{T}$ cells, the predictive value of $\mathrm{CD} 226$ expression for immune-checkpoint therapies, including anti-TIGIT or anti-PD-1 therapy, has been suggested. An immune monitoring study in patients with PDAC revealed that mFOLFIRINOX chemotherapy treatment induces upregulation of CD226 on peripheral-blood $\mathrm{CD} 8^{+} \mathrm{T}$ cells, leading to a positive correlation with antigen-specific $\mathrm{CD} 8^{+} \mathrm{T}$-cell responses after TIGIT or PD-1 blockade, suggesting that a high frequency of $\mathrm{CD} 226^{\text {hi }} \mathrm{CD} 8^{+} T$ cells may improve the response to anti-TIGIT or anti-PD-1 therapy (48). Downregulation of CD226 has also been reported in $T_{\text {reg }}$ cells or $\gamma \delta$ T cells of cancer patients. A high TIGIT/CD226 ratio in $\mathrm{T}_{\text {reg }}$ cells is positively correlated with CD25 ${ }^{\text {hi }}$ Foxp $3^{+} \mathrm{T}_{\text {reg }}$ cell frequencies at tumor sites of melanoma patients and poor clinical outcomes after immune-checkpoint blockade therapies, including anti-PD-1 and/or anti-CTLA4 mAbs (54). In addition, $\mathrm{AML}$ patients who have more $\mathrm{TIGIT}^{+} \mathrm{CD} 226^{-} \gamma \delta$ T cells show lower overall survival rates, suggesting that downregulation of CD226 serves as a novel prognostic biomarker for AML (69).

The role of CD226 signaling in tumor immunity: The importance of the CD226-PVR axis in regulating tumor immunity has been shown in vitro and in vivo in preclinical mouse models. $\mathrm{H}-2^{\mathrm{b}}$-specific $\mathrm{CD} 8^{+} \mathrm{T}$ cells or DX $5^{+} \mathrm{NK}$ cells isolated from CD226 deficient mice are less cytotoxic to PVR-expressing tumor cells, but not to PVR-negative tumor cells (66). Moreover, reduced proliferative capacity of OT-I CD8 ${ }^{+} \mathrm{T}$ cells by deleting CD226 was observed upon stimulation with the ovalbumin (OVA) peptide ${ }^{257-264}$ pulsed T-cell lymphoma cell line EL4 expressing PVR. However, unlike EL4, stimulation with professional antigen-presenting cells (APCs), such as mature BM-derived dendritic cells, did not affect the proliferation of CD226-deficient OT-I CD8 ${ }^{+} \mathrm{T}$ cells, suggesting an essential role of CD226 in promoting effector functions of $\mathrm{CD}^{+} \mathrm{T}$ cells in peripheral tissues, such as tumor cells, where co-stimulatory ligand expressions are limited compared to APCs (70). Consistent with the in vitro results, impaired tumor rejection and survival rates were observed in CD226-deficient mice after transplantation of Meth A tumor cells or injection of chemical carcinogens, including MCA or 7,12-dimethylbenz[a]anthracene (DMBA) (66). After a subcutaneous injection of MC38-OVA tumor cells that triggered a $\mathrm{CD} 8^{+} \mathrm{T}$-cell-mediated antitumor immune response, CD226-deficient mice failed to reject the tumor compared to the wild-type control (70). Impaired NKcell-mediated suppression of tumor growth by CD226 deficiency has been reported in B16/F10 or RM-1 lung-metastases mouse models $(70,71)$. The effect of inhibiting the CD226-PVR axis on antitumor immune responses was further investigated with anti-CD226 blocking mAbs. Unlike the accelerated tumor growth in CD226-deficient mice, blocking CD226 did not affect MC38, CT26, or melanoma tumor growth in wild-type mice $(34,61,72)$. However, administering anti-CD226 mAbs to mice treated with the combination of anti-TIGIT and antiPD-L1 mAbs or anti-PD-1 and anti-GITR mAbs reversed the antitumor effect and survival benefit of the combined treatment, which was accompanied by reduced effector function and frequency of $\mathrm{CD}^{+} \mathrm{T}$ cells at the tumor site $(34,61)$. Although more direct evidence is required to corroborate involvement of the CD226-PVR axis with PD-1 signaling (see above), this effect of CD226 inhibition, not limited to its counterpart TIGIT signaling, suggests that CD226 plays a critical role in antitumor immunity. Molecular mechanistic studies have revealed that CD226 binding to PVR triggers phosphorylation of a tyrosine residue (Y319 in mice; Y322 in humans) in the CD226 ITT-like motif and the corresponding signaling molecules, including Erk, Akt, and p38 leading to activation of T cells and cytotoxicity of NK cells $(48,73)$. The functional importance of CD226 Y322 phosphorylation on the CD8 ${ }^{+} \mathrm{T}$-cell response has been shown by treatment with anti-CD226 agonist mAbs that induce CD226 phosphorylation at Y322. Administering the $\mathrm{CD} 226$ agonistic mAb reinvigorates the dysfunctional $\mathrm{CD} 2266^{\mathrm{lo}} \mathrm{CD} 8{ }^{+} \mathrm{T}_{\mathrm{em}}$ cell response to antigen stimulation, which is linked to increased responsiveness to TIGIT blockade (48).

Most recent studies on CD226 downregulation by EOMES and CBL-B and its impact on antitumor activity of CD8 ${ }^{+} \mathrm{T}$ cells also highlight the crucial role of CD226 for effective cancer immunotherapy $(26,74)$.

Another mechanism of CD226-mediated regulation of tumor immune surveillance is modulating the function and integrity of $T_{\text {reg }}$ cells at the tumor site. $T_{\text {reg }}$ cells isolated from metastatic melanoma patients exhibit a higher suppressive capacity in the presence of anti-CD226 blocking mAbs and PVR-Fc, whereas the anti-TIGIT blocking mAbs treatment exerts opposite effects in $T_{\text {reg }}$ cells after PVR binding (54). A TSDR analysis of the Foxp3 locus revealed that PVR-mediated CD226 activation allowed $T_{\text {reg }}$ cells to acquire effector-like functions that impaired $\mathrm{T}_{\text {reg }}$ cell stability.

\section{CD96}

CD96 is a type I transmembrane glycoprotein of the Ig superfamily that is mainly expressed on T and NK cells. Both mouse and human CD96 interact with PVR, but CD96 binding to Nectin-1 is observed only in mice. Signaling through CD96 has been reported to inhibit the cytotoxicity of NK cells in mouse tumor models, indicating an inhibitory role of CD96. However, whether human CD96 inhibits or activates human NK and T cells needs to be clarified. The CD96 extracellular domain consists of three Ig-like domains (V1, V2/C, and C) and a membrane proximal stalk domain. The CD96 cytoplasmic domain contains a short basic/proline-rich motif and an ITIM-like domain in mice and humans. The human (but not mouse) CD96 cytoplasmic domain contains an YXXM motif, which binds and activates the phosphatidylinositol 3-kinase (PI3K) and AKT pathway (75). The importance of the human 
CD96 YXXM motif in the immune-cell response has not been fully elucidated, because of very low specificity. Human CD96 may function as an activating or inhibiting receptor depending on the cell type and environmental conditions $(21,29,71)$.

CD96 expression and clinical outcomes in cancer: $A$ few studies have shown CD96 expression in TILs from cancer patients. A Cancer Genome Atlas (TCGA) analysis of multiple malignancies revealed that CD96 mRNA expression is highly correlated with T-cell markers, including CD3, CD4, and CD8, whereas the NK-cell marker NCR1 displays only a moderate correlation with CD96 (76). A correlation was observed between CD96 and TIGIT in 22 of 32 tumor types available from the TCGA, and CD96 mRNA was correlated with PD-1 in 12 tumor types (77). No CD96 mRNA expression bias was observed between PD- $1^{\text {hi }}$ and PD- $1^{\text {low }}$ CD8 $8^{+} T$ cells in a single-cell RNA-seq analysis of metastatic melanoma, as was further shown in a CRC TIL analysis by flow cytometry. Multiplexed immunohistochemistry of the tumor tissues from patients with MSI (microsatellite instability)-CRC and melanoma-detected CD96 expression in PD- $1^{+} \mathrm{CD} 8{ }^{+} \mathrm{T}$ cells that accumulated within the tumor parenchyma, whereas most PD- $1^{+} \mathrm{CD} 8^{+} \mathrm{T}$ cells were $\mathrm{CD} 96$ negative (77). Two studies reported opposite observations on the correlation between CD96 expression and clinical outcomes in cancer patients. Sun $\mathrm{H}$ et al. reported that the accumulation of $\mathrm{CD}^{+}{ }^{+} \mathrm{NK}$ cells at the tumor site of HCC patients is associated with poor clinical outcomes (78). In contrast, Peng YP et al. reported that the frequency of $\mathrm{CD}^{+} 6^{+}$or $\mathrm{CD} 226^{+} \mathrm{NK}$ cells was negatively correlated with lymph-node metastasis of pancreatic cancer (79). Because CD96 expression on TILs and its correlation with the clinical response in cancer patients is a critical indicator for the role of CD96 in tumor controls, extensive immune-monitoring studies are required to evaluate CD96 as a therapeutic target for cancer.

Discrepancies of CD96 function in tumor immunity: CD96 was initially characterized as a stimulatory receptor for NK cells (21), but the inhibitory potential of CD96 has been suggested in several mouse-model studies and by CD96 blocking mAbs (75). CD96-deficient mice have better control of tumor growth in MCA-induced fibrosarcoma and B16/F10 lung metastases models than do wild-type mice in an NK-cell-dependent manner that produced increased IFN- $\gamma$ due to a CD96 deficiency (71). CD96 blockade also increases antitumor immune responses of both $\mathrm{CD} 8^{+} \mathrm{T}$ cells and NK cells against B16F10, LWT1 melanoma, 3LL lung carcinoma, RM-1 prostate carcinoma, CT26, and MCA1956 fibrosarcoma cells, and co-blockade of CD96 with anti-TIGIT, anti-PD-1, anti-PD-L1, or anti-CTLA4 more potently inhibits tumor growth $(71,77,80)$. However, recent studies suggest an opposite role for CD96, particularly in the regulation of T-cell responses. Chiang EY et al. reported that CD96 agonism by co-coating with anti-human $\mathrm{CD} 3$ and anti-human CD96 mAb beads (hCD3/hCD96 beads) promotes human $\mathrm{CD}^{+} \mathrm{T}$-cell proliferation similar to that by stimulation with hCD3/hCD28 or hCD3/hOX40 beads (81). Moreover, the effect of CD96 agonism on TCR signaling pathways was shown in the HD-MAR2 T-cell line, which expresses both CD226 and CD96. hCD3/hCD96 bead stimulation increases phosphorylation of ERK and MEK to a greater degree than does stimulation with hCD3/hCD226 beads. Treatment of two distinct CD96 mAbs in a soluble form had no effect on pp65 $5^{495-503}$ specific CD8 ${ }^{+}$ T-cell responses, whereas TIGIT or CD112R blockade increased cytokine production (10), indicating that hCD96 may not act as a co-inhibitory $\mathrm{T}$-cell receptor. It has been speculated that the discrepancies in the role of CD96 in T-cell or NK-cell regulation may come from a difference in the intracellular domain between mCD96 and hCD96, which contains an YXXM motif that is also found in co-stimulatory receptors, such as CD28 and ICOS $(82,83)$. However, Chiang EY et al. provided evidence that mCD96-mPVR binding stimulates $\mathrm{CD} 8^{+} \mathrm{T}$-cell responses as an hCD96-hPVR axis, by employing mCD96 crosslinking to stimulate $\mathrm{CD}^{+} \mathrm{T}$ cells. mCD96 crosslinking with bead-conjugated anti-mCD96 mAbs increases OT-I T-cell killing of OVA SIINFEKL peptide-loaded B16F10 melanoma cells, regardless of mPVR or mNectin-2 expression, whereas administering soluble anti-mCD96 mAbs reduced the killing activity of OT-I T cells by blocking mPVR binding. A more direct effect of mCD96 agonism was shown in the TCR signaling pathway with strong ERK phosphorylation upon stimulation with CD3/ CD96 beads. Contradictory results were observed under the therapeutic tumor setting where both genetic deletion of CD96 and treatment withanti-mCD96 mAbs did not affect tumor growth in CT26 tumor-bearing mice, but rather showed reduced activation of $\mathrm{CD}^{+}{ }^{+}$TILs in the absence of a CD96-PVR interaction (81). This discrepancy in the effect of the CD96 blockade may result from different characteristics of individual anti-CD96 $\mathrm{mAb}$ clones in terms of 1) Fc receptor binding involved in Fc-mediated crosslinking (81), or 2) PVR blocking/non-blocking activities, since it has been previously shown that PVR nonblocker CD96 mAb also increases anti-metastatic activity of NK cells (84). However, more studies are required to demonstrate the role of CD96 in the regulation of antitumor immunity.

\section{CD112R}

$\mathrm{CD} 112 \mathrm{R}$ is an inhibitory immune-checkpoint receptor that is expressed in $\mathrm{CD}^{+}$and $\mathrm{CD}^{+} \mathrm{T}$ cells, $\gamma \delta \mathrm{T}$, NKT, and NK cells. $\mathrm{CD} 112 \mathrm{R}$ is a putative single-transmembrane protein consisting of a single extracellular IgV domain, a transmembrane domain, and a cytoplasmic domain. The CD112R intracellular domain possesses an ITIM-like motif that could be a potential docking site for phosphatases. CD112R inhibits activation of T and NK cells upon interaction with Nectin-2 $(10,27)$. A recent study using preclinical mouse models reported that inhibiting CD112R promotes an antitumor immune response by restoring T-cell activities. Pmel-1 CD8 ${ }^{+}$T cells isolated from Pmel-1 TCR-CD112Rdeficient mice show augmented effector responses, including CD107 expression and effector cytokine production upon gp $100^{25-33}$ stimulation and subsequent co-culture with B16/F10 tumor cells expressing mhgp100 and Nectin-2 (85). Consistent with the in vitro results, MC38 tumor growth was decreased in 
TIGIT family receptors

Hyung-seung Jin and Yoon Park

CD112R-deficient mice in a $\mathrm{CD}^{+}{ }^{+} \mathrm{T}$-cell-dependent manner and exhibited increased effector responses and an inflammatory/cytotoxic gene signature compared with $\mathrm{CD}^{+}{ }^{+}$TILs in wildtype mice (85). Increased IFN- $\gamma$ production by CD112R-deficient $\mathrm{CD} 8^{+}$TILs appeared to be linked to upregulation of PD-L1 in tumor necrosis factor (TNF)- $\alpha$-producing CD11 $b^{+}$myeloid cells that accumulated at the tumor site in CD112Rdeficient mice bearing MC38 tumors. This might be a recapitulation of the adaptive resistance mechanism of immune evasion in cancer patients. Administration of anti-PD-L1 blocking mAbs to established MC38 tumors promotes tumor rejection and survival in CD112R-deficient mice more than in wild-type mice (85). CD112R expression on tumor-infiltrating $C D 8{ }^{+} \mathrm{T}$, $\mathrm{CD}^{+} \mathrm{T}$, and NK cells is found in patients with ovarian, kidney, lung, endometrial, breast, stomach, head and neck, bladder, colorectal, and prostate cancers (10). In particular, $\mathrm{CD}^{+}$and $\mathrm{CD}^{+}{ }^{+}$TILs from lung-cancer patients exhibit higher CD112R expression than that of $\mathrm{T}$ cells from matching normal adjacent tissue. CD112R is also highly expressed on NK cells from a patient with prostate cancer. Moreover, CD112R was co-expressed with TIGIT and PD-1 on CD8 ${ }^{+}$TILs, indicating an exhausted phenotype. Inhibiting CD112R binding to Nectin-2 with an antagonistic mAb-increased IFN- $\gamma$ or IL-2 production in TILs isolated from lung, ovarian, endometrial, head and neck, or kidney cancer patients upon ex vivo co-culture with Mel-624 cells expressing membrane-bound anti-CD3 scFv (Mel-624 OKT3). Co-blockade of CD112R with other immune-checkpoint therapies, including anti-TIGIT or anti-PD-1 mAbs, promoted

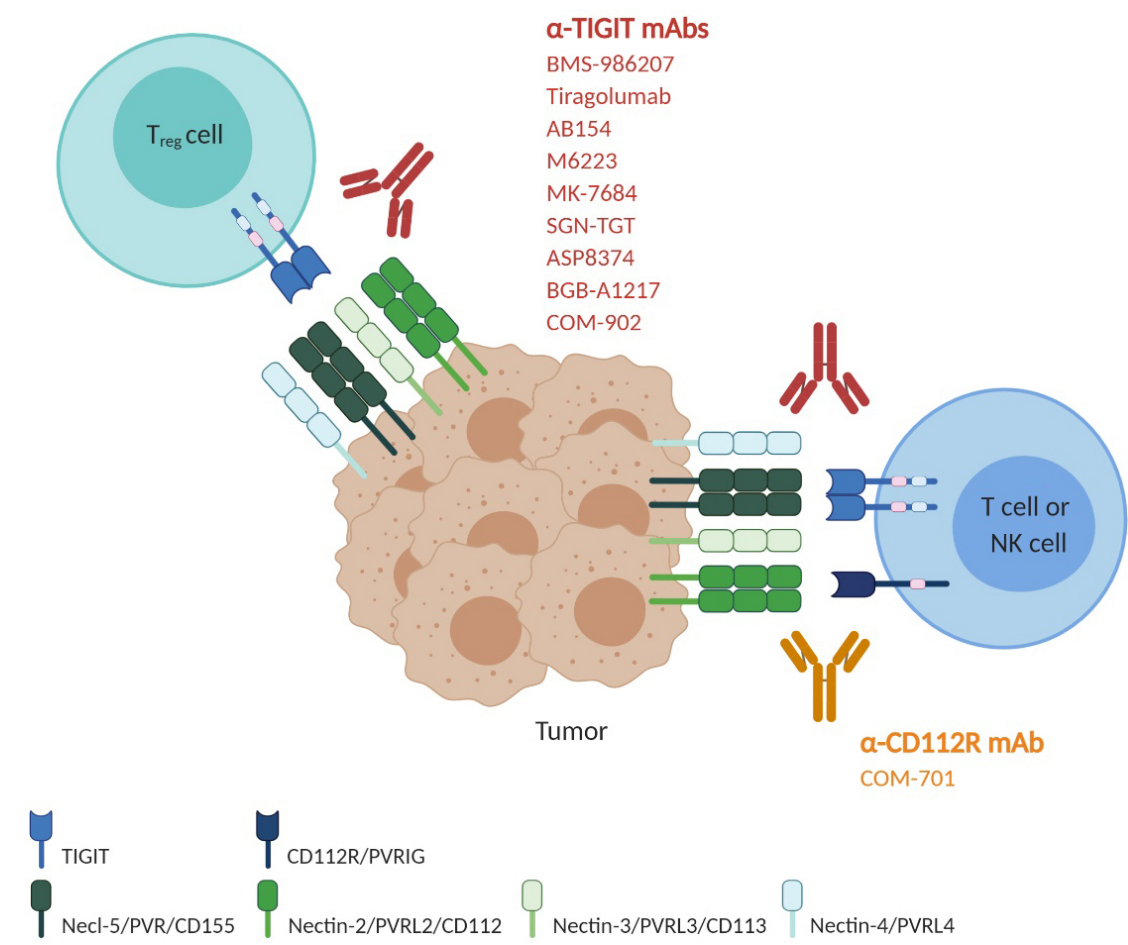

Fig. 2. Blockade of the immune-checkpoint receptors TIGIT and CD112R using antagonistic monoclonal antibodies. Around nine human anti-TIGIT monoclonal antibodies, which have different IgG isotypes or mutant forms, have entered clinical trials. Given that combined blockade by TIGIT and PD-1/PD-L1 exhibited a more powerful anti-tumor effect in preclinical models, many clinical trials are evaluating the safety and efficacy of combination therapy with anti-TIGIT and anti-PD-1/PD-L1 as well as anti-TIGIT monotherapy. The most advanced, anti-TIGIT tiragolumab, is in a phase 3 trial in combination with the anti-PD-L1 atezolizumab and carboplatin and etoposide (CE) for treatment of untreated extensive-stage small-cell lung cancer (NCT04256421). Another phase 3 clinical trial is ongoing for the evaluation of tiragolumab combined with atezolizumab in patients with previously untreated locally advanced unresectable or metastatic PD-L1-selected NSCLC (NCT04294810). Phase I and phase II clinical trials using anti-TIGIT AB154, M6223, MK-7684, SGN-TGT, ASP8374, BGB-A1217 and COM-902, alone or combined with anti-PD-1/PD-L1 antibodies, are ongoing to evaluate the safety and efficacy in patients with metastatic or locally advanced solid tumors (NCT03628677, NCT04262856, NCT04457778, NCT02964013, NCT04165070, NCT04305054 NCT04305041, NCT04303169, NCT04254107 NCT03260322, NCT04047862 and NCT04354246). A phase 1/II clinical trial with anti-TIGIT BMS986207, alone or combined with pomalidomide and dexamethasone, is ongoing in patients with relapsed refractory multiple myeloma who have relapsed after treatment with prior therapies (NCT04150965). There is one anti-CD112R antibody in a clinical trial. COM701 is a humanized anti-CD112R hinge-stabilized IgG4 developed by Compugen. COM701 is being tested in phase 1 clinical trials as monotherapy or in combination with nivolumab or in combination with BMS986207 and nivolumab, in patients with advanced solid tumors, including non-small cell lung cancer, ovarian, breast and endometrial cancer (NCT03667716 and NCT04570839). 
reactivation of $\mathrm{CD}^{+}{ }^{+}$TILs, suggesting that $\mathrm{CD} 112 \mathrm{R}$ and TIGIT may have nonredundant inhibitory signaling modes by dominantly binding to Nectin-2 and PVR, respectively (10). Blocking CD112R and/or TIGIT also increases human NK-cell activation and the trastuzumab-triggered antitumor response (86).

\section{CONCLUDING REMARKS}

Manipulating the interplay between TIGIT family receptors, including TIGIT, CD226, CD112R, CD96 and their ligands, has emerged as an attractive approach for the next generation of cancer immunotherapy that can synergize with PD-1/PD-L1 blockade therapies. TIGIT blockade has demonstrated great promise in preclinical models and has progressed to several ongoing clinical trials in patients with advanced malignancies (Fig. 2). CD112R blockade has also been under clinical evaluation in phase I studies. However, the mechanistic understanding of the regulatory network driven by the TIGIT family receptors, such as receptor dynamics among family members and associated outcomes in the regulation of tumor immunity, is still limited, but it is important to shed light on the still unanswered questions in TIGIT family receptors-based immunotherapies:

1) Whether CD226 activation is associated with the efficacy of the blockades of TIGIT family receptors;

2) Any compensatory expression/mechanism of family members upon TIGIT or CD112R blockade;

3) Any potential synergy of combined treatment of TIGIT family blockade with PD-1/PD-L1 pathway blockade;

4) Any predictive criteria for patient selection in clinical studies of TIGIT or CD112R therapies.

Further mechanistic investigations and translational efforts need to be made within these contexts. These investigations will be crucial for designing effective clinical strategies of ongoing clinical trials and translating other family members into therapeutic interventions.

\section{ACKNOWLEDGEMENTS}

This work was supported by the National Research Foundation of Korea (NRF-2020R1I1A1A01073840 and NRF-2020M3A9G 7103935) and the KIST institutional program. Illustrations were created with BioRender.com.

\section{CONFLICTS OF INTEREST}

The authors have no conflicting interests.

\section{REFERENCES}

1. Thommen DS, Schumacher TN (2018) T cell dysfunction in cancer. Cancer Cell 33, 547-562

2. Chen DS, Mellman I (2013) Oncology meets immunology: the cancer-immunity cycle. Immunity $39,1-10$
3. Ribas A, Wolchok JD (2018) Cancer immunotherapy using checkpoint blockade. Science 359, 1350-1355

4. Gibney GT, Weiner LM, Atkins MB (2016) Predictive biomarkers for checkpoint inhibitor-based immunotherapy. Lancet Oncol 17, e542-e551

5. Zaretsky JM, Garcia-Diaz A, Shin DS et al (2016) Mutations associated with acquired resistance to PD-1 blockade in melanoma. N Engl J Med 375, 819-829

6. Kong X (2018) Discovery of new immune checkpoints: family grows up. Adv Exp Med Biol 1248, 61-82

7. Rotte A, Jin JY, Lemaire V (2018) Mechanistic overview of immune checkpoints to support the rational design of their combinations in cancer immunotherapy. Ann Oncol 29, 71-83

8. Chan CJ, Andrews DM, Smyth MJ (2012) Receptors that interact with nectin and nectin-like proteins in the immunosurveillance and immunotherapy of cancer. Curr Opin Immunol 24, 246-251

9. Sanchez-Correa B, Valhondo I, Hassouneh F et al (2019) DNAM-1 and the TIGIT/PVRIG/TACTILE axis: novel immune checkpoints for natural killer cell-based cancer immunotherapy. Cancers (Basel) 11, 877

10. Whelan S, Ophir E, Kotturi MF et al (2019) PVRIG and PVRL2 are induced in cancer and inhibit CD8(+) T-cell function. Cancer Immunol Res 7, 257-268

11. Marin-Acevedo JA, Soyano AE, Dholaria B, Knutson KL, Lou Y (2006) Cancer immunotherapy beyond immune checkpoint inhibitors. J Hematol Oncol 11, 8

12. Gorvel L, Olive D (2020) Targeting the "PVR-TIGIT axis" with immune checkpoint therapies. F1000Res 9, F1000 Faculty Rev-354

13. Fuchs A, Colonna M (2006) The role of NK cell recognition of nectin and nectin-like proteins in tumor immunosurveillance. Semin Cancer Biol 16, 359-366

14. Nakanishi H, Takai Y (2004) Roles of nectins in cell adhesion, migration and polarization. Biol Chem 385, 885-892

15. Zhang Q, Bi J, Zheng X et al (2018) Blockade of the checkpoint receptor TIGIT prevents NK cell exhaustion and elicits potent anti-tumor immunity. Nat Immunol 19, 723-732

16. Kucan Brlic $P$, Lenac Rovis $T$, Cinamon $G$, Tsukerman $P$, Mandelboim O, Jonjic S (2019) Targeting PVR (CD155) and its receptors in anti-tumor therapy. Cell Mol Immunol $16,40-52$

17. Nishiwada S, Sho M, Yasuda S et al (2015) Clinical significance of CD155 expression in human pancreatic cancer. Anticancer Res 35, 2287-2297

18. Triki H, Charfi S, Bouzidi L et al (2019) CD155 expression in human breast cancer: clinical significance and relevance to natural killer cell infiltration. Life Sci 231, 116543

19. Carlsten M, Norell H, Bryceson YT (2009) Primary human tumor cells expressing CD155 impair tumor targeting by down-regulating DNAM-1 on NK cells. J Immunol 183, 4921-4930

20. Joller N, Hafler JP, Brynedal B et al (2011) Cutting edge: TIGIT has T cell-intrinsic inhibitory functions. J Immunol 186, 1338-1342

21. Fuchs A, Cella M, Giurisato E, Shaw AS, Colonna M (2004) Cutting edge: CD96 (tactile) promotes NK cell-target cell adhesion by interacting with the poliovirus receptor 
(CD155). J Immunol 172, 3994-3998

22. Yu X, Harden K, Gonzalez LC et al (2009) The surface protein TIGIT suppresses $\mathrm{T}$ cell activation by promoting the generation of mature immunoregulatory dendritic cells. Nat Immunol 10, 48-57

23. Stanietsky N, Rovis TL, Glasner A et al (2013) Mouse TIGIT inhibits NK-cell cytotoxicity upon interaction with PVR. Eur J Immunol 43, 2138-2150

24. Deuss FA, Watson GM, Fu Z, Rossjohn J, Berry R (2019) Structural basis for CD96 immune receptor recognition of nectin-like protein-5, CD155. Structure 27, 219-228

25. Okumura G, Iguchi-Manaka A, Murata R, Yamashita-Kanemaru Y, Shibuya A, Shibuya K (2020) Tumor-derived soluble CD155 inhibits DNAM-1-mediated antitumor activity of natural killer cells. J Exp Med 217, 1

26. Weulersse M, Asrir A, Pichler AC et al (2020) Eomesdependent loss of the co-activating receptor CD226 restrains CD8(+) T cell anti-tumor functions and limits the efficacy of cancer immunotherapy. Immunity 53, 824-839

27. Zhu Y, Paniccia A, Schulick AC et al (2016) Identification of CD112R as a novel checkpoint for human T cells. J Exp Med 213, 167-176

28. Pende D, Castriconi R, Romagnani $P$ et al (2006) Expression of the DNAM-1 ligands, nectin-2 (CD112) and poliovirus receptor (CD155), on dendritic cells: relevance for natural killer-dendritic cell interaction. Blood 107, 2030-2036

29. Seth S, Maier MK, Qiu Q et al (2007) The murine pan T cell marker CD96 is an adhesion receptor for CD155 and nectin-1. Biochem Biophys Res Commun 364, 959-965

30. Satoh-Horikawa K, Nakanishi H, Takahashi K (2000) Nectin-3, a new member of immunoglobulin-like cell adhesion molecules that shows homophilic and heterophilic cell-cell adhesion activities. J Biol Chem 275, 10291-10299

31. Reches A, Ophir Y, Stein N et al (2020) Nectin4 is a novel TIGIT ligand which combines checkpoint inhibition and tumor specificity. J Immunother Cancer 8, e000266

32. Harjunpaa $H$, Guillerey $C$ (2020) TIGIT as an emerging immune checkpoint. Clin Exp Immunol 200, 108-119

33. Lozano $E$, Dominguez-Villar $M$, Kuchroo V, Hafler DA (2012) The TIGIT/CD226 axis regulates human T cell function. J Immunol 188, 3869-3875

34. Johnston RJ, Comps-Agrar L, Hackney J et al (2014) The immunoreceptor TIGIT regulates antitumor and antiviral CD8(+) T cell effector function. Cancer Cell 26, 923-937

35. Joller N, Lozano E, Burkett PR et al (2014) Treg cells expressing the coinhibitory molecule TIGIT selectively inhibit proinflammatory Th1 and Th17 cell responses. Immunity 40, 569-581

36. Kurtulus S, Sakuishi K, Ngiow SF et al (2015) TIGIT predominantly regulates the immune response via regulatory T cells. J Clin Invest 125, 4053-4062

37. Yang ZZ, Kim HJ, Wu H et al (2020) TIGIT expression is associated with T-cell suppression and exhaustion and predicts clinical outcome and anti-PD-1 response in follicular lymphoma. Clin Cancer Res 26, 5217-5231

38. Kong Y, Zhu L, Schell TD et al (2016) T-cell immunoglobulin and ITIM domain (TIGIT) associates with CD8+ T-cell exhaustion and poor clinical outcome in AML patients. Clin Cancer Res 22, 3057-3066

39. Chauvin JM, Pagliano O, Fourcade J et al (2015) TIGIT and PD-1 impair tumor antigen-specific CD8(+) T cells in melanoma patients. J Clin Invest 125, 2046-2058

40. Guillerey C, Harjunpaa $\mathrm{H}$, Carrie N et al (2018) TIGIT immune checkpoint blockade restores CD8(+) T-cell immunity against multiple myeloma. Blood 132, 1689-1694

41. He W, Zhang H, Han F et al (2017) CD155T/TIGIT signaling regulates $\mathrm{CD} 8(+)$ T-cell metabolism and promotes tumor progression in human gastric cancer. Cancer Res 77, 6375-6388

42. O'Brien SM, Klampatsa A, Thompson JC et al (2019) Function of human tumor-infiltrating lymphocytes in earlystage non-small cell lung cancer. Cancer Immunol Res 7, 896-909

43. Ostroumov D, Duong S, Wingerath J et al (2020) Transcriptome profiling identifies TIGIT as a marker of T cell exhaustion in liver cancer. Hepatology [Online ahead of print]

44. Stalhammar G, Seregard S, Grossniklaus HE (2019) Expression of immune checkpoint receptors Indoleamine 2,3-dioxygenase and T cell Ig and ITIM domain in metastatic versus nonmetastatic choroidal melanoma. Cancer Med 8, 2784-2792

45. Xu D, Zhao E, Zhu C et al (2020) TIGIT and PD-1 may serve as potential prognostic biomarkers for gastric cancer. Immunobiology 225, 151915

46. Wu L, Mao L, Liu JF et al (2019) Blockade of TIGIT/ CD155 signaling reverses T-cell exhaustion and enhances antitumor capability in head and neck squamous cell carcinoma. Cancer Immunol Res 7, 1700-1713

47. Lucca LE, Lerner BA, Park C et al (2020) Differential expression of the T-cell inhibitor TIGIT in glioblastoma and MS. Neurol Neuroimmunol Neuroinflamm 7, e712

48. Jin HS, Ko M, Choi DS et al (2020) CD226(hi)CD8(+) T cells are a prerequisite for anti-TIGIT immunotherapy. Cancer Immunol Res 8, 912-925

49. McLane LM, Abdel-Hakeem MS, Wherry EJ (2019) CD8 T cell exhaustion during chronic viral infection and cancer. Annu Rev Immunol 37, 457-495

50. Hashimoto $M$, Kamphorst AO, Im SJ et al (2018) CD8 T cell exhaustion in chronic infection and cancer: opportunities for interventions. Annu Rev Med 69, 301-318

51. Tang W, Pan X, Han D et al (2019) Clinical significance of CD8(+) T cell immunoreceptor with Ig and ITIM domains(+) in locally advanced gastric cancer treated with SOX regimen after D2 gastrectomy. Oncoimmunology 8, e1593807

52. Fuhrman CA, Yeh WI, Seay HR et al (2015) Divergent phenotypes of human regulatory $T$ cells expressing the receptors TIGIT and CD226. J Immunol 195, 145-155

53. Duan X, Liu J, Cui J et al (2019) Expression of TIGIT/ CD155 and correlations with clinical pathological features in human hepatocellular carcinoma. Mol Med Rep 20, 3773-3781

54. Fourcade J, Sun Z, Chauvin JM et al (2018) CD226 opposes TIGIT to disrupt Tregs in melanoma. JCI Insight 3, e121157

55. Dixon KO, Schorer $M$, Nevin J et al (2018) Functional anti-TIGIT antibodies regulate development of autoimmunity and antitumor immunity. J Immunol 200, 30003007

56. Chiu DK, Yuen VW, Wing-Sum Cheu J et al (2020) 
Hepatocellular carcinoma cells up-regulate PVRL1, stabilizing poliovirus receptor and inhibiting the cytotoxic T-cell response via TIGIT to mediate tumor resistance to PD1 inhibitors in mice. Gastroenterology 159, 609-623

57. Lee BR, Chae S, Moon J et al (2020) Combination of PD-L1 and PVR determines sensitivity to PD-1 blockade. JCl Insight 5, e128633

58. Jin HS, Choi DS, Ko $M$ et al (2019) Extracellular pH modulating injectable gel for enhancing immune checkpoint inhibitor therapy. J Control Release 315, 65-75

59. Hung AL, Maxwell R, Theodros D et al (2018) TIGIT and PD-1 dual checkpoint blockade enhances antitumor immunity and survival in GBM. Oncoimmunology 7, e1466769

60. Grapin M, Richard C, Limagne E et al (2019) Optimized fractionated radiotherapy with anti-PD-L1 and anti-TIGIT: a promising new combination. J Immunother Cancer 7, 160

61. Wang B, Zhang W, Jankovic V et al (2018) Combination cancer immunotherapy targeting PD-1 and GITR can rescue CD8(+) T cell dysfunction and maintain memory phenotype. Sci Immunol 3, eaat7061

62. Liu S, Zhang H, Li M et al (2013) Recruitment of Grb2 and SHIP1 by the ITT-like motif of TIGIT suppresses granule polarization and cytotoxicity of NK cells. Cell Death Differ 20, 456-464

63. Shibuya K, Lanier LL, Phillips JH (1999) Physical and functional association of LFA-1 with DNAM-1 adhesion molecule. Immunity 11, 615-623

64. Enqvist M, Ask EH, Forslund E et al (2015) Coordinated expression of DNAM-1 and LFA-1 in educated NK cells. J Immunol 194, 4518-4527

65. Shibuya A, Campbell D, Hannum C et al (1996) DNAM-1, a novel adhesion molecule involved in the cytolytic function of T lymphocytes. Immunity 4, 573-581

66. Iguchi-Manaka A, Kai H, Yamashita Y et al (2008) Accelerated tumor growth in mice deficient in DNAM-1 receptor. J Exp Med 205, 2959-2964

67. Sanchez-Correa B, Gayoso I, Bergua JM et al (2012) Decreased expression of DNAM-1 on NK cells from acute myeloid leukemia patients. Immunol Cell Biol 90, 109115

68. Minnie SA, Kuns RD, Gartlan KH et al (2018) Myeloma escape after stem cell transplantation is a consequence of T-cell exhaustion and is prevented by TIGIT blockade. Blood 132, 1675-1688

69. Jin Z, Lan T, Zhao Y et al (2020) Higher TIGIT(+)CD226(-) gammadelta $T$ cells in patients with acute myeloid leukemia. Immunol Invest 1-11 [Online ahead of print]

70. Gilfillan S, Chan Cl, Cella M et al (2008) DNAM-1 promotes activation of cytotoxic lymphocytes by nonprofessional antigen-presenting cells and tumors. J Exp Med 205, 2965-2973

71. Chan CJ, Martinet L, Gilfillan S et al (2014) The receptors CD96 and CD226 oppose each other in the regulation of natural killer cell functions. Nat Immunol 15, 431-438

72. Lakshmikanth T, Burke S, Ali TH et al (2009) NCRs and DNAM-1 mediate NK cell recognition and lysis of human and mouse melanoma cell lines in vitro and in vivo. J Clin Invest 119, 1251-1263

73. Zhang Z, Wu N, Lu Y, Davidson D, Colonna M, Veillette A (2015) DNAM-1 controls NK cell activation via an ITT-like motif. J Exp Med 212, 2165-2182

74. Braun M, Aguilera AR, Sundarrajan A et al (2020) CD155 on tumor cells drives resistance to immunotherapy by inducing the degradation of the activating receptor CD226 in CD8(+) T cells. Immunity 53, 805-823

75. Georgiev H, Ravens I, Papadogianni G, Bernhardt G (2018) Coming of age: CD96 emerges as modulator of immune responses. Front Immunol 9, 1072

76. Lepletier A, Lutzky VP, Mittal D et al (2019) The immune checkpoint CD96 defines a distinct lymphocyte phenotype and is highly expressed on tumor-infiltrating T cells. Immunol Cell Biol 97, 152-164

77. Mittal D, Lepletier A, Madore J et al (2019) CD96 is an immune checkpoint that regulates CD8(+) T-cell antitumor function. Cancer Immunol Res 7, 559-571

78. Sun $H$, Huang Q, Huang $M$ et al (2019) Human CD96 correlates to natural killer cell exhaustion and predicts the prognosis of human hepatocellular carcinoma. Hepatology 70, 168-183

79. Peng YP, Xi CH, Zhu Y et al (2016) Altered expression of CD226 and CD96 on natural killer cells in patients with pancreatic cancer. Oncotarget 7, 66586-66594

80. Blake SJ, Stannard K, Liu J et al (2016) Suppression of metastases using a new lymphocyte checkpoint target for cancer immunotherapy. Cancer Discov 6, 446-459

81. Chiang EY, de Almeida PE, de Almeida Nagata DE et al (2020) CD96 functions as a co-stimulatory receptor to enhance CD8(+) T cell activation and effector responses. Eur J Immunol 50, 891-902

82. Meyer D, Seth S, Albrecht J et al (2009) CD96 interaction with CD155 via its first Ig-like domain is modulated by alternative splicing or mutations in distal Ig-like domains. J Biol Chem 284, 2235-2244

83. Chambers CA (2001) The expanding world of co-stimulation: the two-signal model revisited. Trends Immunol 22, 217-223

84. Roman Aguilera A, Lutzky VP, Mittal D et al (2018) CD96 targeted antibodies need not block CD96-CD155 interactions to promote NK cell anti-metastatic activity. Oncoimmunology 7, e1424677

85. Murter B, Pan X, Ophir E et al (2019) Mouse PVRIG has CD8(+) T cell-specific coinhibitory functions and dampens antitumor immunity. Cancer Immunol Res 7, 244-256

86. Xu F, Sunderland A, Zhou Y, Schulick RD, Edil BH, Zhu Y (2017) Blockade of CD112R and TIGIT signaling sensitizes human natural killer cell functions. Cancer Immunol Immunother 66, 1367-1375 